\title{
Indooraktivitäten und damit zusammenhängende Einrichtungen
}

\author{
Coordinating Lead Authors (CLAs) \\ Ulrike Pröbstl-Haider \\ Lead Authors (LAs) \\ Claudia Hödl, Kai Illing \\ Contributing Authors (CAs) \\ Robert Ranzi, Oliver Fritz
}

\subsection{Allgemeine Entwicklung und Trends}

Der Aufbau und Ausbau von landschaftsunabhängigen Angeboten, wie Wellness- und Indoorsportanlagen, wird häufig in Zusammenhang mit einer Förderung des Ganzjahrestourismus genannt und als Möglichkeit gesehen, die Winter- bzw. Schneeabhängigkeit von Alpendestinationen zu verringern. Solche schneeunabhängigen Angebote scheinen vor allem für Destinationen in tieferen Höhenlagen eine geeignete Möglichkeit zu sein, sich an die veränderten klimatischen Bedingungen anzupassen und eine Saisonverlängerung in den Sommer und Frühherbst hinein zu erzielen (Müller und Weber 2007; Müller et al. 2007; Scott und McBoyle 2007; Siegrist und Gessner 2011; Clivaz et al. 2012).

In diesem Zusammenhang werden insbesondere dem Wellness- und Gesundheitstourismus gute Marktchancen und Potenziale eingeräumt (Schobersberger 2008; BMWi 2011; Adamer-König et al. 2018), wobei eine Abgrenzung der beiden Begriffe nicht immer einfach ist (Smith und Puczkó 2009; Johnston et al. 2011; Voigt und Pforr 2014). Während der Gesundheitstourismus (Medical Tourism) mit dem Ziel der nachweisbaren Verbesserung des Gesundheitszustandes im Urlaub seinen Fokus auf die Behandlung, Linderung und Heilung von Krankheiten legt, beschäftigt sich der Wellnesstourismus mit einer ganzheitlichen Förderung und der Erhaltung von Gesundheit und Wohlbefinden (Voigt und Pforr 2014). In der Praxis verlaufen die Grenzen jedoch nur selten trennscharf und der Wellnesstourismus wird bisweilen als Untersparte des Gesundheitstourismus gesehen (Müller und Lanz Kaufmann 2001; Heublein und Kronthaler 2014; Peters et al. 2017). Auch der Begriff der „Medical Wellness“, die „klassische“ Wellness mit medizinisch-therapeutischen Angeboten kombiniert, unter anderem mit Anti-Aging-Anwendungen und schönheitschirurgischen Behandlungen, zeigt, wie fließend hier die Übergänge in der Realität verlaufen können (Illing
2004; Dörpinghaus 2009; Schletterer Wellness \& Spa Design GmbH 2010). Ein mögliches Hilfsmittel zur Differenzierung stellt das Wellnesskontinuum dar (Ardell 1977; Miller 2005; Berg 2008); im touristischen Kontext wird auch der Begriff Medical-Wellnesskontinuum verwendet. Dieses Modell ermöglicht eine Einteilung der Betriebe, je nachdem, ob sie einen stärkeren Medizin- oder Wellnessbezug aufweisen, und trägt damit der Vielfalt an Betriebstypen Rechnung. Die unterschiedliche Ausrichtung spiegelt sich auch in den verwendeten Begriffen wider, z. B. Medizinhotel, Medical Hotel etc. bei eher medizinisch geprägten Angeboten bzw. Relaxoase, Spa ${ }^{1}$, Wellnesshotel oder dergleichen, wenn Entspannung und Wohlbefinden im Mittelpunkt stehen. Gefördert werden beide Formen durch die älter werdende Gesellschaft in den wichtigsten Herkunftsländern und dem Inlandsmarkt.

Unabhängig von der genauen Definition und Abgrenzung herrscht Einigkeit darüber, dass sich die zunehmende Beliebtheit von Gesundheits- und Wellnessangeboten auf gemeinsame Nenner bzw. globale Megatrends zurückführen lässt (siehe Abschn. 1.2.7).

\subsection{Relevante Entwicklungen in den wichtigsten Herkunftsländern}

\subsubsection{Wellness}

Der Wellnesstourismus ist in Österreich stark von der Nachfrage im Inland geprägt. Laut Erhebungen der Österreich Werbung verbrachten im Tourismusjahr 2017/2018 ca. 8 \%

\footnotetext{
${ }^{1}$ In einem Spa werden, typischerweise unter Zuhilfenahme von (Heil-) Wasser, Fitness, div. Behandlungen und Entspannungsmöglichkeiten angeboten. Zum Ursprung des Begriffs gibt es verschiedene Theorien bzw. Herleitungsversuche (siehe dazu Illing 2015).
} 
der befragten Urlauberinnen und Urlauber einen Wellnessurlaub $^{2}$. Von ihnen stammten wiederum rund $49 \%$ aus Österreich, gefolgt von Deutschland mit $31 \%$ und der Schweiz mit 9 \% (Österreich Werbung 2019a).

Die typische Wellnessurlauberin bzw. der typische Wellnessurlauber in Österreich ist Mitte 40, reist in Begleitung der Partnerin bzw. des Partners (52 \%) und nächtigt in einem 4-Sterne-Hotel (65\%). Die durchschnittliche Aufenthaltsdauer beträgt dabei 5,3 Nächte und die Topaktivitäten stellen Schwimmen bzw. Baden (68 \%) sowie die Nutzung von Wellness- und Schönheitsangeboten (54\%) dar (Österreich Werbung 2019a). Während im Winter der Besuch von Thermen (46\%) und Skifahren (40\%) ebenfalls beliebte Aktivitäten sind, so gewinnen demgegenüber auf das Gesamtjahr bezogen Spazierengehen/Bummeln/Flanieren (52\%) und Wandern (51 \%) an Bedeutung (Österreich Werbung 2018, 2019a).

Neueste Zahlen weisen zudem den Gesundheitstourismus mit einem Nächtigungszuwachs von 3,7\% gegenüber dem Sommer des Vorjahres als eine der momentan am stärksten wachsenden Tourismussparten in Österreich aus. Auch hier spielen mit einem Anteil von 56,5 \% vor allem die Gäste aus dem Inland eine tragende Rolle (Fleischhacker 2019). Auffallend ist die vergleichsweise hohe Aufenthaltsdauer der Gesundheitsurlaubenden, die mit 7,7 Nächten deutlich über dem Wert von 5,3 in der Sparte Wellnessurlaub liegt (Österreich Werbung 2019a, b) und sogar noch deutlicher über dem gesamtösterreichischen Schnitt von 3,3 Nächten (Statistik Austria 2020). Trotz dieser aus wirtschaftlicher Sicht positiven Zahlen darf aber nicht vergessen werden, dass der Anteil an Gesundheitsurlaubenden (Gäste, die in Kurheimen und Rehakliniken genächtigt haben, sind hier ausgenommen) derzeit lediglich $2 \%$ beträgt (Österreich Werbung 2019b). Aus methodischer Sicht muss außerdem angemerkt werden, dass die genannten Nächtigungszahlen auf der speziellen Auswertung ausgewählter Gemeinden mit ortsgebundenen Heilvorkommen, Kuranstalten, Einrichtungen für Kneipp- und sonstige Kuren sowie Thermalquellen und Thermen beruhen (Luftkurorte nicht miteingeschlossen; Fleischhacker 2019) und daher eine Kombination aus Wellness- und Gesundheitstourismus wiedergeben.

Obwohl Wasser nicht zwangsläufig das definierende Element von Wellnesstourismus sein muss, so bilden Spa- und Thermenanlagen wohl das bekannteste Angebot in diesem Bereich (Voigt und Pforr 2014). Die Beliebtheit der Aktivität Schwimmen bzw. Baden bei den österreichischen Wellnessurlaubenden spricht ebenfalls dafür, dass der durchschnittliche Gast den Begriff Wellness mit dem Medium Wasser verbindet und das Vorhandensein entsprechender Anlagen, wie z. B. Pools, Thermalbecken, Hallenbäder, erwartet.

\footnotetext{
2 Samplegröße: $n=1489$ Wellnessurlaubende; Frage: Wie würden Sie Ihren Aufenthalt bezeichnen? (Max. 3 Urlaubsarten können genannt werden).
}

Die zunehmende Nachfrage nach Wellnesstourismus hat mitunter dazu geführt, dass sich eine steigende Zahl an Destinationen als Gesundheits- bzw. Wellnessdestination vermarktet, um auf diese Weise stärker in das Bewusstsein der gewählten Zielgruppe zu rücken und diese gezielt anzusprechen (Voigt und Pforr 2014). Ein entsprechender Versuch, die Alpen als Wellnessdestination zu etablieren, verbirgt sich hinter der Marke „Alpine Wellness“. Diese länderübergreifende Marketingkollaboration ist ein Zusammenschluss alpiner Regionen in Österreich, Deutschland, Südtirol und der Schweiz (IQ MEDIEN GMBH o.J.; Österreich Werbung o.J.; Pechlaner und Fischer 2004; Clivaz et al. 2012). Die ausgewählten „Alpine Wellness Hotels“ werben mit ihrer besonderen Topografie, der natürlichen Landschaft sowie mit den Schlagworten Authentizität, Exklusivität und Luxus. Auf der entsprechenden Webseite ist es außerdem möglich, Hotels zu speziellen Themen zu suchen, beispielsweise in den Kategorien Sportwellness, Romantikwellness, Medical-Wellness, Skiwellness oder Family-Wellness.

Die Entwicklung hin zu solchen Themenhotels, deren spezielles Angebot auf eine bestimmte Zielgruppe abzielt, stellt einen weiteren Trend in der Tourismusbranche dar, um sich von der Konkurrenz abzuheben. Expertinnen und Experten aus dem Tourismus gehen davon aus, dass ein verstärkter Fokus auf Spezialisierung, z. B. im Bereich Medical-Wellness, und Innovation nötig sein wird, um zukünftig bestehen zu können. Das trifft speziell auf den alpinen Raum mit seiner hohen Dichte an Wellnessanlagen und einer stellenweise bereits vorhandenen Marktsättigung zu (Illing 2004; Smith und Puczkó 2009; Schletterer Wellness \& Spa Design GmbH 2010; Peters et al. 2017). Mag das Angebot eines Spabereichs noch vor einigen Jahren einen Wettbewerbsvorteil gegenüber anderen Betrieben bedeutet haben, so gilt in der Praxis heute eine zum Hotelkonzept passende Wellness- und Spaanlage ab einer bestimmten Kategorie, wie der 4- und 5-Sterne-Hotellerie, bereits als Voraussetzung (Heyes et al. 2015).

\subsubsection{Indoorklettern}

Neben der zunehmenden Nachfrage nach gesundheitstouristischen Angeboten und Wellness lässt sich noch ein weiterer für den österreichischen Tourismus relevanter Indoortrend beobachten: das Interesse an Sportklettern und Bouldern. Das Interesse an dieser Aktivität ist in den letzten Jahren stark gestiegen, was zu einer Zunahme von Kletterhallen geführt hat. Der Deutsche Alpenverein (DAV) hat in einer Umfrage beispielsweise festgestellt, dass 2004 noch etwa $18 \%$ seiner Mitglieder Kletterhallen aufgesucht haben, im Jahr 2017 waren es allerdingst bereits $27 \%$ (DAV 2018). Für Österreich fehlen zwar vergleichbare Zahlen, doch auch hierzulande wurden vor allem in den letzten Jahren vermehrt Hallen mit dem Hauptzweck Klettersport gebaut (Lampersberger et al. 
2017a). Vor allem in Tirol ist eine große Zahl an teilweise sehr modernen Kletterhallen zu finden (Tirol Werbung o.J.; Verein Climbers Paradise Tirol o.J.). Möglichkeiten zum Indoorklettern sind aber auch in den übrigen Bundesländern zahlreich vorhanden, nicht zuletzt im urbanen Raum, wie z. B. Wien (ÖAV o.J.). Der Deutsche Alpenverein schätzt die Zahl der deutschen Kletterhallen auf insgesamt ca. 500, mit einem besonders großen Angebot in München, wo sich mit dem Kletterzentrum München-Süd (Thalkirchen) nach eigenen Angaben sogar die weltweit größte Kletterhalle befindet (Burmester 2010; DAV 2018, o.J.).

Insgesamt lag der Anteil der Gäste, die im Sommer 2018 Klettern als ihre Hauptaktivität bezeichnet haben, bei $1 \%$ (von weiteren $3 \%$ wurde Klettern als Nebenaktivität ausgeübt). Die wichtigste Nation unter den Gästen stellte dabei Deutschland mit 49 \% dar (Österreich Werbung 2019c). Zwar können Kletterhallen als vergleichsweise sichere Alternative zu alpinen Klettersteigen angesehen werden, die aufgrund des klimawandelbedingten Tauens von Permafrost und der damit verbundenen erhöhten Gefahr von Steinschlag und/oder Felssturz evtl. in Zukunft nicht oder nur mehr eingeschränkt genutzt werden können (Braun 2009; DAV und ÖAV 2011; BMWFJ 2012; siehe Abschn. 7.3.2). Andererseits sind die österreichischen Berge für $69 \%$ der Klettergäste der Hauptentscheidungsgrund, gefolgt vom Angebot an Wanderwegen $(50 \%)$. Dementsprechend sind Wandern (86\%) und Bergsteigen $(68 \%)$ die von dieser Gruppe am häufigsten genannten Nebenaktivitäten (Österreich Werbung 2019c). Da das Naturerlebnis für dieses Gästesegment offenbar sehr wichtig ist, werden sie mehrheitlich wohl nicht in Kletterhallen ausweichen, sondern, wenn möglich, Regionen aufsuchen, in denen der Sport weiterhin gefahrlos im Freien ausgeübt werden kann. Indoorklettern dürfte für sie nur dann eine Alternative darstellen, wenn die Zahl geeigneter Regionen stark zurückgehen sollte bzw. für den Fall, dass die Anreise mit unverhältnismäßig viel Zeit und Kosten verbunden ist. Für diejenigen Gäste, die Klettern als Nebenaktivität ausüben, kann Indoorklettern aber durchaus eine attraktive wetter- und jahreszeitenunabhängige Ergänzung des lokalen Angebots einer Destination darstellen.

\subsubsection{Indoorschneesport}

Eine weitere Entwicklung, die zwar für Österreich aufgrund der herrschenden geografischen und topografischen Verhältnisse weniger relevant ist als für andere Länder, jedoch immer wieder in den heimischen Medien diskutiert wird und an dieser Stelle daher trotzdem genannt werden soll, sind Skihallen, die ganzjährig genutzt werden können (Scott und McBoyle 2007). Diese sind in mehreren europäischen Ländern, z. B. in Großbritannien, den Niederlanden und in Deutschland, aber auch in Russland, zu finden (Österreich Werbung 2015). In
Österreich wird zwar momentan noch keine solche Indoorskihalle betrieben, jedoch besteht beispielsweise eine Kooperation zwischen der SalzburgerLand Tourismus GmbH und der JEVER Fun Skihalle im deutschen Neuss sowie mit der Skihalle snej.com nahe Moskau. Ziel der Kooperation ist es, die Besucherinnen und Besucher im Ausland auf das heimische Angebot aufmerksam zu machen und damit Werbeeffekte für den Winterurlaub in Salzburg zu erzielen (Österreich Werbung 2015; SalzburgerLand Tourismus GmbH o.J.). Auch andere österreichische Organisationen bzw. Betriebe, wie beispielsweise Kärnten Werbung und Ötztal Tourismus, treten als Sponsoren, Förderer oder Partner von Skihallen im europäischen Ausland auf (Österreich Werbung 2015).

Im Sinne der Nachhaltigkeit und eines sparsamen Umgangs mit Energie, aber auch wegen ihres großen Flächenverbrauchs, der damit verbundenen Versiegelung (Umweltbundesamt $\mathrm{GmbH}$ o.J.) und ihres Einflusses auf das Landschaftsbild können Skihallen jedoch nicht als geeignete Anpassungsstrategie an den Klimawandel in Österreich angesehen werden. Außerdem ist fraglich, ob und inwieweit Skihallen für die Gäste überhaupt ein attraktives Alternativangebot zum alpinen Schneesport darstellen, sind doch Berge, Landschaft und Natur wichtige Entscheidungskriterien für den Urlaub in Österreich (WKO 2018).

\subsection{Einfluss des Klimawandels bzw. Einfluss auf den Klimawandel}

\subsubsection{Einfluss des Klimawandels auf Indooraktivitäten}

Allgemein lässt sich festhalten, dass Indoorangebote in vielen Destinationen lediglich eine Ergänzung zu Outdooraktivitäten darstellen. Ist deren Ausübung durch den Klimawandel gefährdet bzw. nur mehr eingeschränkt möglich, dann wird auch dieses ergänzende Angebot einen entsprechenden Nachfragerückgang nicht verhindern können. Langfristig werden nur diejenigen Angebote attraktiv bleiben, die sich durch eine Unique Selling Proposition (USP) auszeichnen. Diese kann beispielsweise in der Lage eines Wellnesshotels in einer attraktiven (Winter-)Landschaft bestehen (Stichwort „Skiwellness"). Geht diese jedoch infolge des Klimawandels verloren, so werden die betroffenen Betriebe auch ihre USP verlieren und sich neu orientieren müssen (z. B. bei einem Rückgang der Schneefälle in tiefer liegenden Wintersportorten).

Bei einer Zunahme von extremen Wetterereignissen und einer steigenden Steinschlaggefahr könnten Klettersportlerinnen und Klettersportler in Zukunft vermehrt in Hallen ausweichen. Ob dies aufgrund des fehlenden Naturerlebnisses auch auf Touristinnen und Touristen zutrifft oder nur auf regional ansässige (Hobby-)Sportelnde, ist allerdings fraglich (siehe dazu die Ausführungen in Abschn. 8.2.2 und 7.3.1). 
Im Sommer können Wellnessangebote, die auf Hallenund Thermalbädern beruhen, aufgrund der steigenden Temperaturen an Attraktivität verlieren, beispielsweise gegenüber Seen bzw. anderen angebotenen Aktivitäten, wie Golfen, Radfahren, Wandern oder Reiten (Fleischhacker et al. 2009; Formayer und Kromp-Kolb 2009; BMWFJ 2012). Gleichzeitig ist jedoch eine räumliche Verschiebung der Nachfrage in höhere und damit kühlere Lagen bzw. eine Wiederbelebung der klassischen „Sommerfrische“ möglich (Fleischhacker et al. 2009; Abegg und Steiger 2011; APCC 2014) - eine Entwicklung, von der die Sparte der alpinen Wellnessanbieter bei Bereitstellung attraktiver Angebote, die auf die Bedürfnisse „hitzegeplagter" Stadtbewohnerinnen und Stadtbewohner abgestimmt sind, profitieren würde (Jiricka-Pürrer et al. 2018).

\subsubsection{Einfluss von Indooraktivitäten auf den Klimawandel}

Ein Faktor, der bei Indooranlagen besonders ins Gewicht fällt, ist ihr Energiebedarf, der je nach Typ und Bauweise beträchtlich ausfallen kann, aber auch von der Art des Angebots abhängt. Medizinisch ausgerichtete Tourismusbetriebe verfügen zum Beispiel meist über verschiedene Behandlungsräume, deren Raumtemperatur und damit Energiebedarf mit dem jeweiligen therapeutischen Angebot zusammenhängt. Im Unterschied dazu weisen Wellnessbetriebe in der Regel größere Wasserbereiche auf, die besonders energieintensiv sind. Lampersberger et al. (2017a) gehen beispielsweise für ein Indoorhallenbad mit einer Wasserfläche von $700 \mathrm{~m}^{2}$ von einem Referenzwert von $3345 \mathrm{MWh} / \mathrm{a}$ aus; unter den laut Studie untersuchten Sportstättentypen nehmen Hallenbäder damit deutlich den Spitzenwert ein, wohingegen Kletterhallen mit $146 \mathrm{MWh} / \mathrm{a}$ den niedrigsten Energieverbrauch aufweisen. Der vergleichsweise hohe Strom- und Wärmebedarf in der gehobenen Hotellerie (siehe dazu die Ausführungen in Abschn. 4.3.3) wird zwar generell auf das Vorhandensein zusätzlicher Indoorangebote, wie z. B. Saunen, Wellnessbereiche und Schwimmbäder, zurückgeführt, welcher Anteil konkret auf sie entfällt, wird aber nicht angegeben (Bayer et al. 2011).

Zudem gilt es zu beachten, dass langfristiger Erfolg nur durch eine fortlaufende Erneuerung des Angebots gewährleistet werden kann. Neben der Lage und Umgebung spielen die architektonische Gestaltung bzw. das Design für die Zufriedenheit der Kundschaft in der Wellnesssparte eine besonders wichtige Rolle (Lanz Kaufmann 1999; Illing 2004). Wellnesseinrichtungen müssen demzufolge regelmäßig, d. h. in einer Zeitspanne von ca. 5 Jahren, umgestaltet werden, um attraktiv zu bleiben (Lanz Kaufmann 1999; ghh consult GmbH 2010). Zwar sind hierzu keine wissenschaftlichen Studien verfügbar, abhängig von der jeweiligen Nutzungsintensität sowie der Material- und Wartungsqualität, geht die Fachpresse aber davon aus, dass Einrichtungen wie Saunen, Bäder und Dampfbäder bei täglichem Betrieb ca. alle 5-7 Jahre komplett erneuert werden müssen (ghh consult GmbH 2010). Der für Erneuerung und Umgestaltung nötige Energiebedarf muss, neben dem für den laufenden Betrieb, daher bei Überlegungen zum Klimaschutz ebenfalls berücksichtigt werden.

Des Weiteren stellen Indooranlagen auch eine Erhöhung der Versiegelung dar, was sich bei Starkregenereignissen negativ auswirken und im Einzelfall - je nach Gesamtfläche, lokalen Gegebenheiten und herrschendem Versiegelungsgrad - zu Überschwemmungen oder zu einer kurzzeitigen Überlastung des Entwässerungsnetzes beitragen kann (Umweltbundesamt GmbH o.J.).

\subsection{Anpassungs-, Minderungsmaßnahmen und Strategien}

Indoorsportstätten zeichnen sich in vielen Fällen durch einen hohen Energieverbrauch aus, wobei Hallenbäder am energieintensivsten abschneiden. Gleichzeitig gibt es jedoch eine Vielzahl an Möglichkeiten, wie die jeweiligen Gebäudetypen effizienter gestaltet und betrieben werden können (Lampersberger et al. 2017a). Hier soll im Speziellen auf Kletterhallen (als Sonderform von Sporthallen) sowie auf Hallenbäder und damit zusammenhängende Anlagen, wie Ausgleichsbecken, Sanitärbereich, Sauna u. Ä., eingegangen werden. Die folgenden Ausführungen beziehen sich auf den aktuellen Zustand, bei dem sowohl in Sport- bzw. Kletterhallen als auch in Wellnessbädern der Wärmebedarf in der Regel deutlich über dem Strombedarf liegt. Es ist allerdings nicht auszuschließen, dass sich dieses Verhältnis in Zukunft angesichts steigender Temperaturen verändern wird und der Stromverbrauch zunimmt, z. B. zur Klimatisierung der Räume im Sommer (siehe dazu Abschn. 4.4.2), vor allem wenn alter Gebäudebestand mit mobilen Geräten nachgerüstet wird.

\subsubsection{Kletterhallen}

Kletterhallen ähneln in ihrem Energieverbrauch dem von Sporthallen, bei denen der Wärmeverbrauch einen Großteil $(67 \%)$ des Gesamtverbrauchs ausmacht. Eine Besonderheit von Kletterhallen stellt der vergleichsweise hohe Beleuchtungsaufwand dar, da für optimale Bedingungen auch die vertikalen Flächen ausgeleuchtet werden müssen, sowie der etwas geringere Warmwasserbedarf. Maßnahmen zur Energiereduktion sollten daher vorrangig auf die Bereiche Wärmebereitstellung, Belüftung und Beleuchtung fokussieren, da hier die größten Einspareffekte zu erzielen sind (Lampersberger et al. 2017a). Folgende Lösungen sind in diesem Sinne nach Lampersberger et al. (2017a) empfehlenswert: 
- Passivhausbauweise zur Minimierung von Wärmeverlusten über die Gebäudehülle,

- Flächenheizsysteme (z. B. Fußboden- und Wandheizflächen, Deckenstrahlplatten) statt Luftheizungsanlagen,

- Berücksichtigung erneuerbarer Energien im Sinne einer nachhaltigen Wärmeversorgung (z. B. thermische Solaranlagen, Erdwärmepumpen, Biomasseheizkessel),

- Wärmetauscher zur Rückgewinnung von Lüftungswärmeverlusten,

- gezielter Einsatz natürlicher Querlüftung (z. B. mittels automatischer Klappfenster),

- Mischformen aus natürlicher und mechanischer Belüftung,

- effiziente Nutzung von Tageslicht zur Reduktion des Beleuchtungsstrombedarfs,

- zeitgemäßes Beleuchtungskonzept (z. B. Präsenzmelder, Tageslichtsensoren, energieeffiziente Leuchtmittel/ LEDs),

- bei Neubauten Betonkernaktivierung der Bodenplatte zur Schaffung großer thermischer Speicher in Kombination mit solarer Wärmebereitstellung.

Allgemein kann festgehalten werden, dass die meisten Kletterhallen in Österreich bereits einen niedrigen Energieverbrauch pro Anlage aufweisen, was vermutlich darauf zurückzuführen ist, dass sie zum Großteil erst in den letzten Jahren erbaut worden sind und daher höheren Energiestandards entsprechen als Altbauten (Lampersberger et al. 2017a). Aufgrund der eingesetzten Energieinnovationen werden von Lampersberger et al. (2017b) die Kletterhalle Saalfelden $\left(\mathrm{CO}_{2}\right.$-Einsparung: ca. $\left.17 \mathrm{t} / \mathrm{Jahr}\right)$ sowie das Kletterzentrum Innsbruck ( $\mathrm{CO}_{2}$-Einsparung: ca. $\left.87 \mathrm{t} / \mathrm{Jahr}\right)$ dennoch als BestPractice-Beispiele besonders hervorgehoben.

\subsubsection{Spa- und Wellnessanlagen}

Da Hallenbäder ganzjährig beheizt werden müssen sowie das Becken- und Brauchwasser für die Duschen vorgewärmt werden muss, fällt ihr Wärmeverbrauch in der Regel deutlich höher als der Strombedarf aus. Strom wird in erster Linie für Lüftungs- und Pumpenanlagen benötigt sowie für den elektrischen Betrieb von Saunaanlagen. Einspareffekte lassen sich daher in erster Linie durch eine Minimierung der Wärmeverluste erzielen (Lampersberger et al. 2017a). Folgende Maßnahmen können zu einer entsprechenden Senkung des Energieverbrauchs beitragen (Energie Schweiz 2010; Perincioli 2010; BMWFW et al. 2015; Lampersberger et al. 2017a):

- Minimierung von Wärmeverlusten über die Gebäudehülle (z. B. Sicherstellung einer dichten Gebäudehülle, hochwertige Dämmung - insbesondere bei Fenstern und verglasten Bereichen, Vermeidung von Wärmebrücken),
- Berücksichtigung erneuerbarer Energien (z. B. thermische Solaranlagen),

- Wärmerückgewinnung aus dem Abwasser von Becken und Duschen (z. B. mittels Plattenwärmetauscher oder mithilfe statischer Systeme in Kombination mit Wärmepumpen),

- Wärmerückgewinnung aus der Abluft von Lüftungsanlagen mittels Wärmetauscher,

- Verminderung von Wärmeverlusten durch Verdunstung (z. B. Schwimmbadabdeckung, Abdeckung und Dämmung von Ausgleichsbecken, Quergefälle zum Abfließen des Wassers an Hallenböden),

- Senkung der Verdampfungsrate zur Reduktion des Entfeuchtungsaufwandes der Lüftungsanlage (z. B. durch geringfügige Erhöhung der Luftfeuchte, sofern dadurch keine Korrosionsschäden entstehen),

- Reduktion des Wasserverbrauchs im Sanitärbereich (z. B. Zweitastensystem bei WC-Anlagen, Duschtaster mit Zeitschaltfunktion, Einbau von Durchflussbegrenzern),

- Optimierung der Betriebsführung durch

- Anpassung von Wasserumwälzmengen an den Bedarf,

- Temperaturabsenkung außerhalb der Nutzungszeiten,

- bedarfsgesteuerte Anpassung der Luftwechselrate und der Entfeuchtung von Lüftungsanlagen,

- Festlegen von Betriebszeiten bzw. Zeitsteuerung für Wellnessanlagen und Attraktionen statt durchgängigen Betriebs (z. B. für Whirlpool, Sauna, Sprudelliegen/Sprudelbad, Gegenstromanlage, Wasserfälle),

- effiziente Nutzung von Tageslicht zur Reduktion des Beleuchtungsstrombedarfs,

- zeitgemäßes Beleuchtungskonzept (z. B. Präsenzmelder im Sanitärbereich, Tageslichtsensoren, energieeffiziente Leuchtmittel/LEDs).

Zur effizienten Analyse und Optimierung empfiehlt sich, neben dem Vorhandensein fachkundigen Personals als Grundvoraussetzung, außerdem die Einführung eines Energieverbrauchsmonitorings (BMWFW et al. 2015; Lampersberger et al. 2017a).

Als Best-Practice-Beispiele im Bereich Wellness, die sich durch besondere Energieinnovationen auszeichnen, werden von Lampersberger et al. (2017b) die Aqua Nova bei Wiener Neustadt ( $\mathrm{CO}_{2}$-Einsparung: ca. $\left.220 \mathrm{t} / \mathrm{Jahr}\right)$ sowie die Wellnessoase Hummelhof in Linz ( $\mathrm{CO}_{2}$-Einsparung: ca. $\left.48 \mathrm{t} / \mathrm{Jahr}\right)$ genannt.

Bei fachgerechtem Einsatz des Baustoffes Holz können auch Schwimmbäder bzw. Wellnesseinrichtungen in Passivhausbauweise errichtet werden, wie das Schwimmbad im Hotel Edelweiss in Wagrain sowie das 1. Kärntner Badehaus in Millstatt zeigen. Neben den ökologischen Vorzügen von Holz sprechen auch Baubiologie, Statik und Kosten für seine Verwendung. Im Innenausbau wirkt sich Massivholz, insbesondere Zirbenholz, zudem positiv auf das Wohlbefinden 
aus, was speziell für Wellnessanlagen relevant ist (z. B. im Saunabereich oder in Ruheräumen; Ronacher et al. 2013).

\section{Thermen}

Eine Sonderstellung unter den Spa- und Wellnessanlagen nehmen touristisch genutzte Thermalquellen ein, da hier die Möglichkeit besteht, diese direkt in die Energiegewinnung einer Region einzubinden.

Tiefengeothermie findet in Österreich hauptsächlich im Oberösterreichischen Molassebecken, gefolgt vom Steirischen Becken sowie zu einem geringeren Anteil im Wiener Becken mit der Niederösterreichischen Molassezone und teilweise auch im Pannonischen Becken statt. Vor allem in der Steiermark stehen diese Projekte infolge ihrer Entstehungsgeschichte oft in direktem Zusammenhang mit balneologisch genutzten Thermalquellen (Goldbrunner 2010, 2015; Haslinger et al. 2016). Hydrothermale Energie wird insbesondere zu Heizzwecken genutzt, bei ausreichender Wassertemperatur und -menge kann technisch aber auch Strom erzeugt werden. Bei entsprechenden Heizsystemen reichen relativ geringe Vorlauftemperaturen aus, sodass eine mehrstufige Nutzung der Wärmeenergie möglich ist (Amt der Oö. Landesregierung et al. 2012). In Bad Waltersdorf (Stmk) wird das geförderte und über $60^{\circ} \mathrm{C}$ heiße, mineralisierte Wasser beispielsweise zuerst an lokale Fernwärmeabnehmer geliefert, bevor es durch die Heiltherme genutzt wird (z. B. zur Warmwasserbereitung, Wärmeversorgung der Lüftungsanlagen, Warmhaltung der Becken, Fußbodenheizung). Danach steht das auf nunmehr ca. $38^{\circ} \mathrm{C}$ abgekühlte Wasser als Heilwasser zum Baden in den Thermalwasserbecken zur Verfügung. In einem letzten Schritt der Kaskade wird das Wasser zur Heizung des mit der öffentlichen Heiltherme verbundenen Quellenhotels verwen$\operatorname{det}$ (OTVG o.J.; APA-OTS 2014). Eine ähnliche Doppel- bzw. Mehrfachnutzung des Thermalwassers findet beispielsweise auch in Bad Blumau (Stmk) sowie in Bad Schallerbad (Oö) und Geinberg (Oö) statt (Goldbrunner 2010, 2015).

Vorteilhaft ist, dass Tiefengeothermie eine kontinuierliche und bedarfsgerechte Versorgung mit erneuerbarer Energie ermöglicht und grundsätzlich mit niedrigen Betriebskosten verbunden ist. Nachteilig sind hingegen die hohen Investitionskosten, die komplexe Geologie Österreichs sowie das Risiko hydrochemischer Reaktionen des Thermalwassers (z. B. Lösungs- und Fällungsprozesse sowie Korrosionsvorgänge), die den Betrieb beeinflussen sowie zusätzliche Kosten verursachen können. Ein möglicher Schritt, die Attraktivität hydrothermaler Geothermie dennoch zu erhöhen bzw. das vorhandene Potenzial auszuschöpfen, ${ }^{3}$ liegt u. a. in der Kostenoptimierung von Bohrungen sowie in einer Vereinfachung der notwendigen Genehmigungsverfahren (Könighofer et al. 2014; Weiss et al. 2014).

\footnotetext{
${ }^{3}$ Bezogen auf die energetische Nutzung, ein Ausbau der balneologischen Nutzung ist hier nicht gemeint, da die Dichte an Thermalbädern, speziell in Ostösterreich, ohnehin bereits hoch ist.
}

\subsection{Handlungsoptionen, Kommunikations- und Forschungsbedarf}

\subsubsection{Ansatzpunkte für Maßnahmen, die Überwindung von Barrieren und Kooperation}

Die Angebotsdiversifizierung ist zwar ein wichtiger Schritt der Klimawandelanpassung, das damit verbundene Potenzial darf aber nicht überschätzt werden (Fleischhacker et al. 2009). Ein Beleg dafür ist beispielsweise, dass ein Ersatzprogramm an skifreien Tagen, wie z. B. die Nutzung von Wellnessangeboten, zwar für viele Gäste infrage kommt, ein solches bei einem einwöchigen Urlaub jedoch durchschnittlich an max. 3 Tagen akzeptiert wird (Pröbstl et al. 2008). Anstatt auf energie- und kostenaufwendige Investitionen zu setzen, bietet sich daher vor allem im Wellnessbereich das Eingehen von Kooperationen mit anderen Anbietern in der Destination an. Die einfachste und am weitesten verbreitete Kooperationsform stellt dabei die Öffnung der hoteleigenen Wellnessinfrastruktur für externe Gäste bzw. für Gäste benachbarter Betriebe dar (Lanz Kaufmann 1999; Formayer und Kromp-Kolb 2009). Von diesem Schritt können vor allem kleinere Unternehmen profitieren, die nicht über die notwendigen Ressourcen verfügen, um ihren Gästen selbst entsprechende Anlagen anzubieten. Konkurrenzvorteile können stattdessen aber auch durch die Erweiterung des Know-hows und innovative Dienstleistungsangebote erzielt werden (Lanz Kaufmann 1999). Wichtig für die Wellnessurlaubenden ist dabei vor allem die Freundlichkeit des Personals sowie die Servicequalität (Illing 2004; GfK Travelscope 2018).

Eine weitere Anpassungsmöglichkeit stellt eine Weiterentwicklung des Angebots dar, weg von Wasser als das definierende Element und stattdessen hin zu einem ganzheitlichen Wellnessbegriff (Voigt und Pforr 2014). Außerdem kann versucht werden, das bestehende Angebot durch Outdooraktivitäten zu ergänzen und auf diese Weise die Größe der notwendigen Innenanlagen und damit auch den Energiebedarf zu reduzieren. In Österreich bietet sich in diesem $\mathrm{Zu}$ sammenhang der Ausbau spezifischer Aspekte der ,alpinen Wellness“" an (z. B. Luft, Höhenlage, Licht, Ernährung und Kultur; Pechlaner und Fischer 2004; Müller und Weber 2007; Müller et al. 2007; Müller und Weber 2008). Vor allem Kulinarik bzw. das Thema gesunde Ernährung sowie Angebote zur persönlichen Weiterentwicklung können dabei eine wichtige Rolle spielen. Auch der Fokus auf Familien und Vertreterinnen und Vertreter der Generation Y (Millennials) sowie der Generation 50+ als (zukünftig) stark wachsende Gästegruppen erscheint derzeitigen Prognosen zufolge lohnenswert (Illing 2004; Rulle 2004; Wellness Tourism Worldwide 2011; Dorn-Petersen 2015; GfK Travelscope 2018; Veres et al. 2018). 


\subsubsection{Ansatzpunkte für die Akzeptanz von Maßnahmen, Information und Bewusstseinsbildung}

Der große Energieverbrauch, der Spa- und Wellnessanlagen zugeschrieben wird (Bohdanowicz und Martinac 2007; Formayer und Kromp-Kolb 2009; Gössling et al. 2012), ist - im Gegensatz zu dem Aufwand, der für die Pistenbeschneiung betrieben wird - nicht sichtbar, wird in den Medien nicht thematisiert und ist im öffentlichen Bewusstsein daher auch nicht präsent. Dadurch entstehen Verzerrungen in der Wahrnehmung der Klimaauswirkungen der verschiedenen Urlaubsformen, die in weiterer Folge die Nachfrage beeinflussen und sich negativ auf das Image von Destinationen (Echtner und Ritchie 2003; Ritchie und Crouch 2003) und bestimmten Freizeitaktivitäten auswirken können. grischconsulta (2012) geben zwar an, dass der Stromverbrauch pro Gast und Skitag in der Schweiz lediglich 5,3 kWh beträgt und damit deutlich unter einem Besuch der Therme Meran in Südtirol $(14,7 \mathrm{kWh})$ oder des Schweizer Hallenbades Zernez $(20,8 \mathrm{kWh})$ liegt. Im österreichischen Skigebiet Kanzelwand/Fellhorn benötigt ein Gast allerdings laut eigenen Angaben des Gebiets insgesamt rund $18 \mathrm{kWh}$ pro Skitag (Oberstdorf Kleinwalsertal Bergbahnen o.J.). Um einer Fehlinformation bzw. Verwirrung der Urlauberinnen und Urlauber entgegenzuwirken und einen objektiven Vergleich zu ermöglichen, bedarf es daher der Erhebung und Kommunikation entsprechender verlässlicher Kennzahlen (BMK o.J.).

Ein weiterer wichtiger Schritt besteht darin, den Gästen vor Augen zu führen, dass vor allem das Anreiseverhalten bzw. die Wahl des Urlaubsortes einen entscheidenden Beitrag zur Reduktion negativer Umweltauswirkungen im Tourismus leisten kann (Juvan und Dolnicar 2017; siehe Kap. 3). Gleichzeitig gilt es, auf Angebotsseite den Energieverbrauch so weit wie möglich zu reduzieren und diese Bemühungen auch den Gästen zu vermitteln, um auf diese Weise die Akzeptanz von ansonsten evtl. auf Unverständnis stoßenden Maßnahmen zu erhöhen (z. B. eingeschränkte Betriebszeiten oder Zeitschaltung bestimmter Attraktionen im Spa- und Wellnessbereich). Bewährte Instrumente zur Energie- und damit auch Kosteneinsparung, aber auch zur Kommunikation der erbrachten Umweltbemühungen stellen die Einführung eines betrieblichen Umweltmanagementsystems, zertifiziert nach ISO 14001:2015 oder EMAS (Bernard und Voss 2012), bzw. eines Energiemanagementsystems dar (siehe Abschn. 4.1.) oder die Verpflichtung zur Einhaltung bestimmter Umweltstandards mit entsprechendem Label (z. B. Österreichisches Umweltzeichen für Tourismus und Freizeitwirtschaft oder EU Ecolabel).

\subsubsection{Wissenslücken und Forschungsbedarf}

Daten zur Reaktion von Urlaubenden auf Folgeeffekte des Klimawandels fehlen für viele Tourismusbereiche, daher können aufgrund dieser fehlenden Faktenlage meist nur MutmaBungen bzw. vorsichtige Prognosen angestellt werden. Beispielsweise haben Befragungen in Kletterhallen zwar gezeigt, dass die meisten Nutzerinnen und Nutzer ihrem Hobby auch im Freien nachgehen (Burmester 2010; Hindinger 2010). Ob Kletterhallen für diese Gruppe allerdings auch im Umkehrschluss eine Alternative zu Klettersteigen sind und alpine Destinationen, die über entsprechende Indooranlagen verfügen, weiterhin attraktive Urlaubsorte darstellen, ist nicht bekannt. Vor dem Hintergrund dessen, dass es Kletternde mit steigender Erfahrung bevorzugt in die freie Natur zieht (Hindinger 2010), erscheint dies jedoch unwahrscheinlich. Eine weitere Wissenslücke besteht darin, wie sich steigende Temperaturen im Sommer auf die Nachfrage im Wellness- und Thermentourismus auswirken. Da die Mehrzahl der Gäste bisherigen Untersuchungen zufolge allerdings nur begrenzt Bereitschaft zeigt, ihr Urlaubsverhalten zu ändern (Fleischhacker et al. 2009; Clivaz et al. 2012), ist mit entsprechenden Ausweichprozessen zu rechnen (z. B. Aufsuchen von Regionen, wo Klettern im Freiland gefahrlos möglich ist, Buchung von Wellnessurlauben in höheren Lagen mit niedrigeren Lufttemperaturen).

\subsection{Zusammenfassung}

Im Indoorbereich ist für Österreich vor allem der Gesundheits- und Wellnesstourismus relevant, dem allgemein große Marktchancen und Potenziale eingeräumt werden und der sich durch wachsende Nächtigungszahlen auszeichnet (hohe Übereinstimmung, starke Beweislage). Regional ist stellenweise aber bereits eine Marktsättigung bzw. ein sehr dichtes Angebot mit entsprechender Konkurrenz zwischen den Anbietern vorhanden. Spezialisierung und Innovation sind in Zukunft daher besonders wichtig, wie z. B. Themenhotels mit Fokus auf eine bestimmte Zielgruppe, Schaffung ganzheitlicher Wellnessangebote, stärkere Einbindung von Kulinarik und Ausbau nichtwasserbasierter Aktivitäten (hohe Übereinstimmung, mittlere Beweislage).

Da sich Wellnessanlagen durch einen hohen Ressourcenverbrauch auszeichnen, sind Einsparungen in diesem Bereich besonders relevant, vor allem die Minimierung von Wärmeverlusten, aber auch die Verminderung des Wasserund Stromverbrauchs (hohe Übereinstimmung, mittlere Beweislage). Die technischen Möglichkeiten dazu sind, wie mehrere Best-Practice-Beispiele zeigen, durchaus vorhanden. Entsprechende Anreize und finanzielle Unterstützung können vonseiten der Politik über Förderungen geschaffen werden, aber auch konkrete Zielvorgaben auf freiwilliger 
Basis (z. B. Kriterienkatalog für energieeffiziente Wellnessbetriebe) bis bin zu verbindlichen Standards sind wichtige Instrumente.

Durch den Klimawandel kann in Zukunft vor allem der Bereich „alpine Wellness“ im Sinne eines Revivals der klassischen „Sommerfrische“ profitieren, dazu müssen von den Betrieben aber auch entsprechende Angebote geschaffen bzw. Packages geschnürt werden (hohe Übereinstimmung, schwache Beweislage). Im Winter dient Wellness zwar als Ergänzungsprogramm im Skiurlaub, sie kann diesen aber nicht ersetzen; das dahin gehende Potenzial darf also nicht überbewertet werden (hohe Übereinstimmung, mittlere Beweislage). Die Relevanz bzw. das Marktpotenzial anderer Indooraktivitäten für den heimischen Tourismus (z. B. Indoorklettern in Hallen) lässt sich derzeit nicht abschätzen, es wird aber als vergleichsweise gering eingestuft.

\section{Kernaussagen - Kapitel 8}

- Der Gesundheits- und Wellnesstourismus wird für die Zukunft als wichtiger Wachstumsmarkt gesehen (hohe Übereinstimmung, starke Beweislage), die mit den touristischen Angeboten in Zusammenhang stehenden Anlagen zeichnen sich jedoch durch einen hohen Energiebedarf aus (hohe Übereinstimmung, mittlere Beweislage).

- Möglichkeiten zur Einsparung von Treibhausgasemissionen, wie durch Gebäudesanierung, alternative Heizformen oder durch die Nutzung von Abwärme und weitere technische Maßnahmen, aber auch die Unterstützung der Betriebe und die Schaffung von Standards zur Sicherstellung eines möglichst sparsamen Verbrauchs haben eine hohe Bedeutung (hohe Übereinstimmung, schwache Beweislage).

- Wie sich der Klimawandel auf bestimmte Aktivitäten, z. B. Klettern in Hallen oder den Besuch von Thermen, auswirken wird, ist schwer abzuschätzen. Wahrscheinlich ist jedoch, dass ein Ausweichen der Gesundheitsund Wellnessurlauberinnen und -urlauber in Höhenlagen mit niedrigeren Lufttemperaturen auftreten wird (hohe Übereinstimmung, schwache Beweislage).

- Die Anbieter ,alpiner Wellness“ können vom Wiederaufleben der klassischen „Sommerfrische“ infolge des Klimawandels profitieren (hohe Übereinstimmung, schwache Beweislage). Um am Markt erfolgreich zu sein, sind allerdings eine Differenzierung des Angebots - weg von dem starken Fokus auf das Thema Wasser und hin zu einem ganzheitlicheren Wellnessbegriff - sowie Spezialisierung (z. B. auf eine bestimmte Zielgruppe) und Innovation (z. B. Schaffung kreativer Angebote und Packages) notwendig (hohe Übereinstimmung, mittlere Beweislage).

\section{Literatur}

Abegg, B. \& Steiger, R. (2011) Will Alpine summer tourism benefit from climate change? A review. In: Borsdorf, A., Stötter, J. \& Veuillet, E. (Hrsg.) Managing Alpine future II: inspire and drive sustainable mountain regions (IGF-Forschungsberichte, Band 4), S. 268-277. Verlag der Österreichischen Akademie der Wissenschaften (ÖAW), Wien, Österreich. Online unter: https://www.zobodat.at/pdf/IGFForschungsberichte_4_0268-0277.pdf (letzter Zugriff: 01.06.2020).

Adamer-König, E., Illing, K. \& Amort, F.M. (2018) Demographie und Epidemiologie als Determinanten des Gesundheitstourismus 2030. In: Heise, P. \& Axt-Gadermann, M. (Hrsg.) Sport und Gesundheitstourismus 2030:Wie die „Generation plus “ den Markt verändert, S. 33-50. Springer Gabler, Wiesbaden, Deutschland. DOI: https:// doi.org/10.1007/978-3-658-16076-0_3

Amt der Oö. Landesregierung, BMLFUW, LfU Bayern \& StMUV Bayern (Hrsg.) (2012) Grundsatzpapiere zur Thermalwassernutzung im niederbayerisch-oberösterreichischen Molass. Amt der Oö. Landesregierung, Bundesministerium für Land- und Forstwirtschaft, Umwelt und Wasserwirtschaft (BMLFUW), Bayerisches Landesamt für Umwelt (LfU) und Bayerisches Staatsministerium für Umwelt und Gesundheit (StMUV). Online unter: https://www.lfu.bayern.de/ wasser/thermische_nutzung/doc/thermalwasser_grundsatzpapier.pdf (letzter Zugriff: 01.06.2020).

APA-OTS (2014) $100 \%$ grüne Energie für die Heiltherme Bad Waltersdorf. APA-OTS Originaltext-Service GmbH, Wien, Österreich. Online unter: https://www.tourismuspresse.at/presseaussendung/ TPT_20140514_TPT0008/100-gruene-energie-fuer-die-heilthermebad-waltersdorf-bild (letzter Zugriff: 01.06.2020).

APCC (2014) Österreichischer Sachstandsbericht Klimawandel 2014 (AAR14). Austrian Panel on Climate Change (APCC), Verlag der Österreichischen Akademie der Wissenschaften (ÖAW), Wien, Österreich. Online unter: http://www.austriaca.at/APCC_AAR2014.pdf (letzter Zugriff: 24.03.2020).

Ardell, D.B. (1977) High level wellness: an alternative to doctors, drugs, and disease. Rodale Press, Emmaus, PA, USA.

Bayer, G., Sturm, T. \& Hinterseer, T. (2011) Kennzahlen zum Energieverbrauch in Dienstleistungsgebäuden. Gefördert durch den Klimaund Energiefonds im Rahmen des Programms „Neue Energien 2020“. Österreichische Gesellschaft für Umwelt und Technik ÖGUT, Wien, Österreich. Online unter: https:/www.oegut.at/downloads/ pdf/e_kennzahlen-ev-dlg_zb.pdf (letzter Zugriff: 01.06.2020).

Berg, W. (2008) Gesundheitstourismus und Wellnesstourismus. Oldenbourg, München, Deutschland.

Bernard, S. \& Voss, K. (2012) Energieverbrauch in der Hotellerie: zunehmende Bedeutung für Ressourcen- und Klimaschutz. DBZ Spezial 10/2012, 38-41. Online unter: https://www.dbz.de/ download/1243687/DBZ_Hotelspezial_2012.pdf (letzter Zugriff: 01.06.2020).

BMK (o.J.) Ist nachhaltiger Tourismus messbar? Bundesministerium für Klimaschutz, Umwelt, Energie, Mobilität, Innovation und Technologie (BMK), Wien, Österreich. Online unter: https://www.umweltzeichen.at/de/tourismus/nachhaltiger-tourismus/ist-nachhaltigertourismus-messbar (letzter Zugriff: 01.06.2020).

BMWFJ (2012) Klimawandel und Tourismus in Österreich 2030: Auswirkungen, Chancen \& Risiken, Optionen \& Strategien (StudienKurzfassung). Bundesministerium für Wirtschaft, Familie und Jugend (BMWFJ), Wien, Österreich. Online unter: https://www.wko. at/branchen/tourismus-freizeitwirtschaft/hotellerie/130318_Klimawandel_u. Tourismus_in_Oe. 2030_Kurzfassung.pdf (letzter Zugriff: 01.06.2020).

BMWFW, WKO \& ÖHV (2015) Energiemanagement in der Hotellerie und Gastronomie (Leitfaden, 3. überarbeitete Auflage). Bundesministerium für Wissenschaft, Forschung und Wirtschaft (BMWFW), Wirtschaftskammer Österreich (WKO) und Österreichische Hotelier- 
vereinigung (ÖHV), Wien, Österreich. Online unter: https://www. wko.at/branchen/tourismus-freizeitwirtschaft/hotellerie/Energiemanagement-in-Hotellerie-und-Gastronomie.pdf (letzter Zugriff: 28.05.2020).

BMWi (2011) Innovativer Gesundheitstourismus in Deutschland: Leitfaden. Bundesministerium für Wirtschaft und Technologie (BMWi), Berlin, Deutschland. Online unter: https://www.projectm.de/sites/ default/files/2016-04/Leitfaden Gesundheitstourismus.pdf (letzter Zugriff: 01.06.2020).

Bohdanowicz, P. \& Martinac, I. (2007) Determinants and benchmarking of resource consumption in hotels: case study of Hilton International and Scandic in Europe. Energy and Buildings 39(1), 82-95. DOI: https://doi.org/10.1016/j.enbuild.2006.05.005

Braun, F. (2009) Sommer-Bergtourismus im Klimawandel: Szenarien und Handlungsbedarf am Beispiel des hochalpinen Wegenetzes. Dissertation, Universität für Bodenkultur Wien, Österreich. Online unter: https://zidapps.boku.ac.at/abstracts/download.php?dataset_ id=7618\&property id=107 (letzter Zugriff: 24.05 .2020 ).

Burmester, S. (2010) Plastikboom: Immer mehr Kletterhallen. Motor Presse Stuttgart GmbH \& Co. KG, Stuttgart, Deutschland. Online unter: https://www.klettern.de/community/vertical-life/kletterhallenboom.380272.5.htm (letzter Zugriff: 01.06.2020).

Clivaz, C., Doctor, M., Gessner, S., Ketterer, L., Luthe, T., Schuckert, M., Siegrist, D. \& Wyss, R. (2012) Adaptionsstrategien des Tourismus an den Klimawandel in den Alpen: Ergebnisse des alpenweiten Projekts ClimAlpTour in der Schweiz. HSR Hochschule für Technik Rapperswil, Schweiz. Online unter: https:/www.seco.admin.ch/dam/ seco/de/dokumente/Standortfoerderung/Tourismus/Strategische $\% 20$ Themen/Klimawandel/Adaption_Klimawandel_Alpen_ClimAlpTour_2012.pdf.download.pdf (letzter Zugriff: 01.06.2020).

DAV (2018) Klettern in Deutschland - Zahlen, Daten \& Fakten. Deutscher Alpenverein e. V. (DAV), München, Deutschland. Online unter: https://www.alpenverein.de/der-dav/presse/hintergrund-info/ klettern-in-deutschland-zahlen-daten-fakten_aid_31813.html (letzter Zugriff: 01.06.2020).

DAV (o.J.) Kletteranlagen in München und Umgebung. Sektion München des Deutschen Alpenvereins e. V. (DAV), München, Deutschland. Online unter: https:/www.alpenverein-muenchen-oberland. de/bergsport/klettern\#horizontalTab4 (letzter Zugriff: 26.02.2019).

DAV \& ÖAV (2011) Wegehandbuch der Alpenvereine. Deutscher Alpenverein e. V. (DAV), München, Deutschland und Österreichischer Alpenverein (ÖAV), Innsbruck, Österreich. Online unter: https:// www.tirol.gv.at/fileadmin/themen/sport/berg-und-ski/downloads berg_und_ski/berg_und_ski_2012/Alpenverein-Wegehandbuch.pdf (letzter Zugriff: 01.06.2020).

Dorn-Petersen, H. (2015) Zielgruppe Generation Y: Wellness von morgen. hotel consult Unternehmensberatung, Seeon, Deutschland. Online unter: https://www.standort-tirol.at/data.cfm?vpath=ma-old import/downloads/04_dorn-petersen (letzter Zugriff: 01.06.2020).

Dörpinghaus, S. (2009) Medical Wellness: Zukunftsmarkt mit Hindernissen. Forschung Aktuell No. 06/2009 Institut Arbeit und Technik (IAT), Fachhochschule Gelsenkirchen, Deutschland. Online unter: https:/www.econstor.eu/bitstream/10419/57238/1/690101554.pdf (letzter Zugriff: 01.06.2020).

Echtner, C.M. \& Ritchie, J.R.B. (2003) The meaning and measurement of destination image. Journal of Tourism Studies 14(1), 37-48. Online unter: http://citeseerx.ist.psu.edu/viewdoc/download?doi=10.1.1.89.3276\&rep=rep1\&type=pdf (letzter Zugriff: 01.06.2020).

Energie Schweiz (2010) Leitfaden Fit- und Wellnessanlagen: Infoblatt Wellnessbad, Energie- und Kosteneffizienz. Bundesamt für Energie BFE, Ittigen, Schweiz. Online unter: http://www.hotelpower.ch/sites/ default/files/leitfaden_fit-und_wellnessbetrieb_07_infoblatt_wellnessbad.pdf (letzter Zügriff: 02.06.2020).

Fleischhacker, V. (2019) Der Sommertourismus in Österreich 2018: Tendenzen der Nachfragesegmente. ITR - Institut für touristische Raumplanung Ges.m.b.H., Tulln an der Donau, Österreich.
Fleischhacker, E., Formayer, H., Seisser, O., Wolf-Eberl, S. \& KrompKolb, H. (2009) Auswirkungen des Klimawandels auf das künftige Reiseverhalten im österreichischen Tourismus am Beispiel einer repräsentativen Befragung der österreichischen Urlaubsreisenden. BOKU-Met Report 19. Im Auftrag des Bundesministeriums für Wirtschaft, Familie und Jugend (BMWFJ), Wien, Österreich. Online unter: https://meteo.boku.ac.at/report/ (letzter Zugriff: 05.05.2020).

Formayer, H. \& Kromp-Kolb, H. (2009) Klimawandel und Tourismus in Oberösterreich. Im Auftrag der Oberösterreichischen Landesregierung und Oberösterreich Tourismus. BOKU-Met Report 18. Online unter: https://meteo.boku.ac.at/report/BOKU-Met_Report_18_online.pdf (letzter Zugriff: 24.05.2020).

GfK Travelscope (2018) Wellness-Trends 2018. GfK SE, Nürnberg, beauty $24 \mathrm{GmbH}$, Berlin und Wellness-Hotels \& Resorts $\mathrm{GmbH}$, Düsseldorf, Deutschland. Online unter: https://media.wellnesshotelsresorts.de/media/docs/wellness-trends-2018-grafiken1.pdf (letzter Zugriff: 01.06.2020).

ghh consult GmbH (2010) Wirtschaftlichkeit und Rentabilität von Wellnessbereichen in Hotels. In: FORUM Zeitschriften und Spezialmedien GmbH (Hrsg.) Hotelbau - Fachzeitschrift für Hotelimmobilien-Entwicklung (Sonderausgabe 2016: wirtschaftliche Wellnesswelten). Online unter: https://www.hotelbau.de/download/ downloadarchiv/Serie-Wirtschaftliche-Wellnesswelten.pdf (letzter Zugriff: 02.06.2020).

Goldbrunner, J. (2010) Austria - country update. Proceedings World Geothermal Congress 2010in Bali, Indonesien, 25-29 April 2010. Online unter: https://www.geothermal-energy.org/pdf/IGAstandard/ WGC/2010/0134.pdf (letzter Zugriff: 02.06.2020).

Goldbrunner, J. (2015) Austria - country update. Proceedings World Geothermal Congress 2015in Melbourne, Australien, 19-25 April 2015. Online unter: https://openei.org/w/images/c/c4/AUST3.pdf (letzter Zugriff: 02.06.2020).

Gössling, S., Peeters, P., Hall, C.M., Ceron, J.-P., Dubois, G., Lehmann, L.V. \& Scott, D. (2012) Tourism and water use: supply, demand, and security. An international review. Tourism Management 33(1), 1-15. DOI: https://doi.org/10.1016/j.tourman.2011.03.015

grischconsulta (2012) Sind Beschneiungsanlagen die umweltfreundlichen Stromerzeuger von morgen? Medienmitteilung vom 23. November 2012. grischconsulta AG, Chur, Schweiz. Online unter: https://www.grischconsulta.ch/wp-content/uploads/2013/02/Pressemeldung-vom-23.11.2012.pdf (letzter Zugriff: 02.06.2020).

Haslinger, E., Goldbrunner, J., Dietzel, M., Leis, A., Boch, R., Elster, D., Fröschl, H., Gold, M., Hippler, D., Knauss, R., Plank, O., Shirbaz, A. \& Wyhlidal, S. (2016) NoScale: Charakterisierung von thermalen Tiefengrundwässern zur Verhinderung von Ausfällungen und Korrosionen bei Geothermieanlagen. Publizierbarer Endbericht zum Projekt NoScale, gefördert durch den Klima- und Energiefonds im Rahmen des Programms ,e!Mission.at“. Online unter: https://www. energieforschung.at/assets/project/final-report/NoScale-843827Publizierbarer-Endbericht-final.pdf (letzter Zugriff: 02.06.2020).

Heublein, T. \& Kronthaler, F. (2014) Gute Marktchancen und Potenziale für den Gesundheitstourismus in der Schweiz. Die Volkswirtschaft: Das Magazin für Wirtschaftspolitik 9, 48-51. Online unter: https:// dievolkswirtschaft.ch/content/uploads/2014/10/20_Kronthaler Heublein_DE_low.pdf (letzter Zugriff: 02.06.2020).

Heyes. A., Beard, C. \& Gehrels, S. (2015) Can a luxury hotel compete without a spa facility? Opinions from senior managers of London's luxury hotels. Research in Hospitality Management 5(1), 93-97. DOI: https://doi.org/10.1080/22243534.2015.11828332

Hindinger, F. (2010) Trends und Handlungsbedarf im Klettersport - eine empirische Untersuchung. Masterarbeit am Institut für Landschaftsentwicklung, Erholungs- und Naturschutzplanung (ILEN), Universität für Bodenkultur Wien, Österreich. Abstract online unter: https:// abstracts.boku.ac.at/oe_list.php?paID $=3 \&$ paLIST $=0 \&$ paSID $=7954$ (letzter Zugriff: 02.06.2020). 
Illing, K. (2004) „Medical Wellness“ in Hotels und Kliniken: Erfolgsvoraussetzungen für Selbstzahlermedizin. Spektrum Freizeit 26(2), 13 36. Online unter: https://duepublico2.uni-due.de/servlets/MCRFileNodeServlet/duepublico_derivate_00035149/05_illing_204.pdf (letzter Zugriff: 02.06.2020).

Illing, K. (2015) Gesundheit und Freizeit: Institutionen im Wandel. In: Freericks, R. \& Brinkmann, D. (Hrsg.) Handbuch Freizeitsoziologie, S. 587-617. Springer VS, Wiesbaden, Deutschland. DOI: https://doi. org/10.1007/978-3-658-01520-6_24

IQ MEDIEN GMBH (o.J.) Alpine Wellness Hotels. IQ MEDIEN GMBH, Abersee, Österreich. Online unter: https://www.alpine-wellness-hotels.com/ (letzter Zugriff: 02.06.2020).

Jiricka-Pürrer, A., Brandenburg, C., Weber, F. \& Liebl, U. (2018) REFRESH! Revival der Sommerfrische: Inspirationen zur Angebotsentwicklung und Vermarktung für Tourismusverantwortliche in Gemeinden und Regionen. Gefördert durch den Klima- und Energiefonds im Rahmen des Programms „Austrian Climate Research Programme“ (ACRP). Universität für Bodenkultur Wien, Umweltbundesamt Österreich und Hochschule Luzern. Online unter: https:// sommerfrische-neu.boku.ac.at/pdf/01-Anbotsenwicklung.pdf (letzter Zugriff: 02.06.2020).

Johnston, K., Puczkó, L., Smith, M. \& Ellis, S. (2011) Wellness tourism and medical tourism: where do spas fit? Global Spa Summit LLC, New York, NY, USA. Online unter: http://www.espalibrary.eu/media/filer_public/e6/59/e6594eac-cc0e-4cb7-9c7e-080661dba9cd/ gss 2011_wellness tourism_and_medical_tourism.pdf (letzter $\mathrm{Zu}$ griff: 02.06.2020).

Juvan, E. \& Dolnicar, S. (2017) Drivers of pro-environmental tourist behaviours are not universal. Journal of Cleaner Production 166, 879-890. DOI: https://doi.org/10.1016/j.jclepro.2017.08.087

Könighofer, K., Domberger, G., Gunczy, S., Hingsamer, M., Pucker, J., Schreilechner, M., Amtmann, J., Goldbrunner, J., Heiss, H.P., Füreder, J., Burgstaller, G. \& Pölzl, U. (2014) Potenzial der Tiefengeothermie für die Fernwärme- und Stromproduktion in Österreich. Publizierbarer Endbericht zum Projekt GeoEnergie2050, gefördert durch den Klima- und Energiefonds im Rahmen des Programms „Neue Energien 2020“. Online unter: https://www.energieforschung. at/assets/project/downloads/834451-Endbericht-GeoEnergie205030062014-final.pdf (letzter Zugriff: 02.06.2020).

Lampersberger, P., Benke, G., Grim, M., Hüttler, W., Preßmair, G., Schwarz-Viechtbauer, K. \& Szeywerth, F. (2017a) EnergieFit: Innovative Energietechnologien für Sportstätten. Teil 1: Leitfaden für Entscheidungsträger. Gefördert durch den Klima- und Energiefonds im Rahmen des Programms „Energieforschungsprogramm“. Online unter: https://www.klimafonds.gv.at/wp-content/uploads/ sites/6/Leitfaden-EntscheidungstrgerEnergieFit.pdf (letzter $\mathrm{Zu}$ griff:02.06.2020).

Lampersberger, P., Benke, G., Grim, M., Hüttler, W., Preßmair, G., Schwarz-Viechtbauer, K. \& Szeywerth, F. (2017b) EnergieFit: Innovative Energietechnologien für Sportstätten. Teil 3: Sammelband Best Practice. Gefördert durch den Klima- und Energiefonds im Rahmen des Programms „Energieforschungsprogramm“. Online unter: https://www.klimafonds.gv.at/wp-content/uploads/sites/6/Sammelband-Best-PracticeEnergieFit.pdf (letzter Zugriff: 02.06.2020).

Lanz Kaufmann, E. (1999) Wellness-Tourismus: Marktanalyse und Qualitätsanforderungen für die Hotellerie - Schnittstelle zur Gesundheitsförderung. Dissertation, Forschungsinstitut für Freizeit und Tourismus (FIF) der Universität Bern, Schweiz.

Miller, J.W. (2005) Wellness: the history and development of a concept. Spektrum Freizeit 27(1), 84-106. Online unter: https://duepublico2.uni-due.de/servlets/MCRFileNodeServlet/duepublico_derivate_00035061/11_miller_1_05.pdf (letzter Zugriff: 02.06.2020).

Müller, H. \& Lanz Kaufmann, E. (2001) Wellness tourism: Market analysis of a special health tourism segment and implications for the hotel industry. Journal of Vacation Marketing 7(1), 5-17. DOI: https://doi.org/10.1177/135676670100700101
Müller, H. \& Weber, F. (2007) Klimaänderung und Tourismus: Szenarienanalyse für das Berner Oberland 2030. Forschungsinstitut für Freizeit und Tourismus (FIF) der Universität Bern, Schweiz. Online unter: https://www.raonline.ch/pages/edu/pdf5/FIF_BOclimate0407. pdf (letzter Zugriff: 02.06.2020).

Müller, H. \& Weber, F. (2008) 2030: Der Schweizer Tourismus im Klimawandel. Schweiz Tourismus, Bern, Schweiz. Online unter: https://www.seco.admin.ch/dam/seco/de/dokumente/Standortfoerderung/Tourismus/Strategische\%20Themen/Klimawandel/Klimawandel_2030_ST.pdf.download.pdf (letzter Zugriff: 02.06.2020).

Müller, H., Weber, F. \& Thalmann, E. (2007) Tourismus. In: OcCC \& ProClim (Hrsg.) Klimaänderung und die Schweiz 2050: erwartete Auswirkungen auf Umwelt, Gesellschaft und Wirtschaft, S. 79-94. Beratende Organ für Fragen der Klimaänderung (OcCC) und ProClim - Forum für Klima und Global Change, Bern, Schweiz. Online unter: http://www.occc.ch/pdf/291.pdf (letzter Zugriff: 02.06.2020).

ÖAV (o.J.) Kletteranlagen. Österreichischer Alpenverein, Innsbruck, Österreich. Online unter: https://www.alpenverein.at/portal/wGlobal/ content/finder/kletteranlagen.php (letzter Zugriff: 02.06.2020).

Oberstdorf Kleinwalsertal Bergbahnen (o.J.) Wie viel Energie benötigt ein Skigebiet? Fellhornbahn GmbH, Kleinwalsertaler Bergbahn AG, Nebelhornbahn-Aktiengesellschaft, Oberstdorfer Bergbahn AG und Skiliftgesellschaft links der Breitach GmbH \& Co KG. Online unter: https://www.ok-bergbahnen.com/service/mymountainnature/wieviel-energie-benoetigt-ein-skigebiet.html (letzter Zugriff: 02.06.2020).

Österreich Werbung (o.J.) „Alpine Wellness“ - Der Gipfel des Wohlbefindens. Online unter: https://www.austria.info/de/aktivitaeten/ wellness/wellnesshotels-und-wellnesswochenende\#alpine-wellnessder-gipfel-des-wohlbefindens (letzter Zugriff: 02.06.2020).

Österreich Werbung (2015) TIP: Skihallen. Trends in Progress aus der Tourismusforschung. Zur Verfügung gestellt von Österreich Werbung, Wien, Österreich.

Österreich Werbung (2018) T-MONA Kurzinfo Winterurlauber in Österreich: Wellness-/Schönheitsurlauber. T-MONA Urlauberbefragung Winter 2017/2018. Zur Verfügung gestellt von Österreich Werbung, Wien, Österreich.

Österreich Werbung (2019a) T-MONA Kurzinfo Urlauber in Österreich: Wellness-/Schönheitsurlauber. T-MONA Urlauberbefragung 2017/2018. Zur Verfügung gestellt von Österreich Werbung, Wien, Österreich.

Österreich Werbung (2019b) T-MONA Kurzinfo Urlauber in Österreich: Gesundheitsurlauber. T-MONA Urlauberbefragung 2017/2018. Zur Verfügung gestellt von Österreich Werbung, Wien, Österreich.

Österreich Werbung (2019c) T-MONA Kurzinfo Sommerurlauber in Österreich: Kletterer. T-MONA Urlauberbefragung Sommer 2018. Zur Verfügung gestellt von Österreich Werbung, Wien, Österreich.

OTVG (o.J.) Geothermie Bad Waltersdorf. Oststeirische Thermalwasserverwertungsgesellschaft m.b.H., Bad Waltersdorf, Österreich. Online unter: http://members.aon.at/otvg/ (letzter Zugriff: 02.06.2020).

Pechlaner, H. \& Fischer, E. (2004) Alpine Wellness - Auf dem Weg von der Kernkompetenz zum Produkt. In: Bieger, T., Laesser, C. \& Beritelli, P. (Hrsg.) Jahrbuch der Schweizerischen Tourismuswirtschaft 2003/2004, S. 265-283. IDT-HSG, St. Gallen, Schweiz. Online unter: https://www.alexandria.unisg.ch/13843/1/IDT_Torismus_def.pdf (letzter Zugriff: 02.06.2020).

Perincioli, L. (2010) Energiemanagement in der Hotellerie. Bundesamt für Energie BFE und Hotelleriesuisse, Bern, Schweiz. Online unter: http://www.hotelpower.ch/sites/default/files/energie_mgt_hotellerie 2006.PDF (letzter Zugriff: 02.06.2020).

Peters, M., Pikkemaat, B., Gurgiser, W., Mailer, M., Tischler, S., Stotten, R., Schermer, M., Abegg, B. \& Steiger, R. (2017) Bleibt alles anders? Tourismus 2025, Projektendbericht. Bundesministerium für Wissenschaft, Forschung und Wissenschaft (BMWFW), Wien, Österreich. Online unter: https://www.bmlrt.gv.at/service/publikationen/tourismus/studie-tourismus-2025.html (letzter Zugriff: 02.06.2020). 
Pröbstl, U., Dallhammer, E., Formayer, H., Grabler, K., Haas, P., Jesch, M., Krajasits, C., Kulnig, A., Prutsch, A., Stanzer, G. (2008) Strategien zur nachhaltigen Raumentwicklung von Tourismusregionen unter dem Einfluss der globalen Erwärmung am Beispiel der Wintersportregion um Schladming. Endbericht des Projektes STRATEGE. Zitiert in: APCC (2014) Österreichischer Sachstandsbericht Klimawandel 2014 (AAR14), S. 968. Austrian Panel on Climate Change (APCC), Verlag der Österreichischen Akademie der Wissenschaften (ÖAW), Wien, Österreich. Online unter: http://www.austriaca.at/ APCC_AAR2014.pdf (letzter Zugriff: 02.06.2020).

Ritchie, J.R.B. \& Crouch, G.I. (2003) The competitive destination: a sustainable tourism perspective. CABI, Wallingford, Vereinigtes Königreich.

Ronacher, H., Pabinger, P., Meisslitzer, M., Brühwasser, J. \& Weissengruber, P. (2013) Energieeffiziente Schwimmbäder: Pflichtenheft für Planung und Betrieb von energieeffizienten Schwimmbädern \& Wellnesseinrichtungen durch innovative Techniken. Publizierbarer Endbericht zum Projekt Energieeffiziente Schwimmbäder, gefördert durch den Klima- und Energiefonds im Rahmen des Programms „Neue Energien 2020“. Online unter: https://www.architekten-ronacher.at/projects/hotel-edelweiss/publizierbarer-endbericht-furhomepage.pdf (letzter Zugriff: 02.06.2020).

Rulle, M. (2004) EduWellness: vom passiven Konsum zum selbstverantwortlichen Handeln. Spektrum Freizeit 26(2), 53-59. Online unter: https://duepublico2.uni-due.de/servlets/MCRFileNodeServlet/ duepublico_derivate_00035151/07_rulle_204.pdf (letzter Zugriff: 02.06.2020).

SalzburgerLand Tourismus GmbH (o.J.) Alpenpark Neuss: Strategische Partnerschaft im wichtigsten Herkunftsmarkt Deutschland. SalzburgerLand Tourismus $\mathrm{GmbH}$, Hallwang, Österreich. Online unter: https://unternehmen.salzburgerland.com/ slt/partner-kooperationen/nachwuchsschmiede-skihalle/? $\mathrm{ga}=2.55586219 .1674509291 .1591113589-314416245.1591113589$ (letzter Zugriff: 02.06.2020).

Schletterer Wellness \& Spa Design GmbH (2010) Spa trends 2020. Schletterer Wellness \& Spa Design GmbH, Strass im Zillertal, Österreich. Online unter: https://www.ttr.tirol/sites/default/files/2017-10/ Schletterer\%20Spa\%20Trends\%202020.pdf (letzter Zugriff: 27.01.2019).

Schobersberger, W. (2008) Climate change as a chance for Alpine health tourism? In: Borsdorf, A., Stötter, J. \& Veuillet, E. (Hrsg.) Managing Alpine future II: inspire and drive sustainable mountain regions (IGF-Forschungsberichte, Band 4), S. 175-181. Verlag der Österreichischen Akademie der Wissenschaften (ÖAW), Wien, Österreich. Online unter: https://www.zobodat.at/pdf/IGF-Forschungsberichte_2_0175-0181.pdf (letzter Zugriff: 02.06.2020).

Scott, D. \& McBoyle, G. (2007) Climate change adaptation in the ski industry. Mitigation and Adaptation Strategies for Global Change 12(8), 1411. DOI: https://doi.org/10.1007/s11027-006-9071-4

Siegrist, D. \& Gessner, S. (2011) Klimawandel: Anpassungsstrategien im Alpentourismus. Ergebnisse einer alpenweiten Delphi-Befragung. Tw Zeitschrift für Tourismuswissenschaft 3(2), 179-194. DOI: https://doi.org/10.1515/tw-2011-0207

Smith, M. \& Puczkó, L. (2009) Health and wellness tourism. Butterworth-Heinemann, Oxford, Vereinigtes Königreich.

Statistik Austria (2020) Ankünfte, Nächtigungen sowie durchschnittliche Aufenthaltsdauer nach Bundesländern (1995 bis 2019). Bundesanstalt Statistik Österreich, Wien, Österreich. Online unter: https:// www.statistik.at/web_de/statistiken/wirtschaft/tourismus/beherbergung/ankuenfte_naechtigungen/030029.html (letzter Zugriff: 02.06.2020)

Tirol Werbung (o.J.) Kletter- \& Boulderhallen in Tirol. Tirol Werbung $\mathrm{GmbH}$, Innsbruck, Österreich. Online unter: https://www.tirol.at/ reisefuehrer/sport/klettern/kletterhallen (letzter Zugriff: 02.06.2020).

Umweltbundesamt GmbH (o.J.) Flächeninanspruchnahme. Umweltbundesamt GmbH, Wien, Österreich. Online unter: http://www. umweltbundesamt.at/umweltsituation/raumordnung/rp_flaecheninanspruchnahme/ (letzter Zugriff: 02.06.2020).

Verein Climbers Paradise Tirol (o.J.) Kletter- und Boulderhallen in Tirol: Indoor-Vergnügen das ganzeJahrüber.Verein Climbers Paradise Tirol, Imst, Österreich. Online unter: https://www.climbers-paradise.com/ kletterdisziplinen/kletterhallen/?gclid=Cj0KCQjw1MXpBRDjAR I s A H t d N - 1 F 4 y g y 187 e f 0 w 2 n G G v N T I y M QYic6nn4hLAodQx0IjkbFxhmBmSWYQaAjohEALw_wcB (letzter Zugriff: 02.06.2020).

Veres, S., Raich, M. \& Blank, C. (2018) Bedürfnisse und Ansprüche der Silver Ager in der gehobenen Hotellerie am Beispiel Tirols. In: Müller, J. \& Raich, M. (Hrsg.) Die Zukunft der Qualitativen Forschung: Herausforderungen für die Wirtschafts-, Gesundheits- und Sozialwissenschaften, S. 121-140. Springer Gabler, Wiesbaden, Deutschland. DOI: https://doi.org/10.1007/978-3-658-23504-8_7

Voigt, C. \& Pforr, C. (2014) Wellness tourism from a destination perspective. Why now? In: Voigt, C. \& Pforr, C. (Hrsg.) Wellness tourism: a destination perspective, S. 3-18. Routledge, London, Vereinigtes Königreich.

Weiss, W., Fink, C., Haslinger, W., Strasser, C., Wörgetter, M., Monsberger, M., Zottl, A., Fleckl, T., Könighofer, K., Domberger, G., Schreilechner, M. \& Gunczy, S. (2014) Forschung und Innovation für Heizen und Kühlen mit Erneuerbaren: Positionspapier. Berichte aus Energie- und Umweltforschung 28/2014. Bundesministerium für Verkehr, Innovation und Technologie (BMVIT), Wien, Österreich. Online unter: https://nachhaltigwirtschaften.at/resources/iea_pdf/positionspapier_201428_fti_heating_and_cooling.pdf (letzter Zugriff: 02.06.2020)

Wellness Tourism Worldwide (2011) 4WR: Wellness for whom, where and what? Wellness tourism 2020. Full research report. Erstellt von Xellum Ltd., Budapest, Ungarn für Wellness Tourism Worldwide LLC (WTW). Online unter: https://www.globalwellnesssummit. com/wp-content/uploads/Industry-Research/Global/2011-wellness-tourism-worldwide-wellness-for-whom.pdf (letzter Zugriff: 02.06.2020).

WKO (2018) Tourismus und Freizeitwirtschaft in Zahlen: österreichische und internationale Tourismus- und Wirtschaftsdaten, 54. Ausgabe, Juni 2018. Wirtschaftskammer Österreich (WKO), Bundessparte Tourismus und Freizeitwirtschaft, Wien, Österreich. Online unter: https://www.wko.at/branchen/tourismus-freizeitwirtschaft/ tourismus-freizeitwirtschaft-in-zahlen-2018.pdf (letzter Zugriff: 28.05.2020). 
Open Access Dieses Buch wird unter der Creative Commons Namensnennung 4.0 International Lizenz (http://creativecommons.org/licenses/by/4.0/deed.de) veröffentlicht, welche die Nutzung, Vervielfältigung, Bearbeitung, Verbreitung und Wiedergabe in jeglichem Medium und Format erlaubt, sofern Sie den/die ursprünglichen Autor(en) und die Quelle ordnungsgemäß nennen, einen Link zur Creative Commons Lizenz beifügen und angeben, ob Änderungen vorgenommen wurden.

Die in diesem Buch enthaltenen Bilder und sonstiges Drittmaterial unterliegen ebenfalls der genannten Creative Commons Lizenz, sofern sich aus der Abbildungslegende nichts anderes ergibt. Sofern das betreffende Material nicht unter der genannten Creative Commons Lizenz steht und die betreffende Handlung nicht nach gesetzlichen Vorschriften erlaubt ist, ist für die oben aufgeführten Weiterverwendungen des Materials die Einwilligung des jeweiligen Rechteinhabers einzuholen. 